\section{Photocatalytic gold recovery from spent cyanide plating bath solutions}

\author{
Rafael van Grieken*, José Aguado, \\ María-José López-Muñoz and \\ Javier Marugán \\ Department of Chemical and Environmental \\ Technology, Rey Juan Carlos University, \\ C/ Tulipán s/n 28933 Móstoles (Madrid), SPAIN. \\ Fax: 3491488 7068; Tel: 3491488 7007; \\ E-mail: rafael.vangrieken@urjc.es
}

\begin{abstract}
A new procedure is presented for the photocatalytic treatment of the highly stable gold-cyanide complexes remaining in spent plating bath solutions. The process allows the recovery of gold by photocatalytic reduction, increasing simultaneously the availability of cyanide for removal treatment. Gold recovery requires a non-oxidant atmosphere and the presence of hydroxyl radical scavengers. The use of mixed $\mathrm{TiO}_{2} / \mathrm{SiO}_{2}$ photocatalysts leads to an improvement in the gold recovery rate.
\end{abstract}

\section{Keywords}

Photocatalysis; Gold recovery; Cyanide; Plating bath

\section{Introduction}

Gold-cyanide compounds represent the gold-containing chemicals most widely used in mining and electroplating industries. Despite many efforts focused on the substitution of cyanide salts by less toxic compounds, no successful alternatives have been found. The unique complexing capabilities of cyanide ions in aqueous solutions make these compounds the best choice for the heap leaching process in the gold mining sites or for gold plating applications (1).

Wastewater effluents of these processes show significant concentrations of gold-cyanide complexes (2) that, due to its high stability, are very difficult to remove using the conventional oxidation technologies for cyanide degradation (3). Recovery of the gold present in the spent plating bath solutions, a desirable process from an economical standpoint, is currently carried out mainly by electrolysis and ion exchange processes.

Some research groups (4-8) have shown the viability of the photochemical deposition and recovery of metallic gold from aqueous solutions of chloride species like $\mathrm{AuCl}_{3}$ and $\mathrm{AuCl}_{4}^{-}$in the presence of titania. These species are considered easy to reduce, due to their reduction potential of $\mathrm{E}^{0}=+1.0 \mathrm{~V} \mathrm{NHE}$ (NHE, normal hydrogen electrode), whereas the value of the reduction potential in oxygenated systems for $\mathrm{Au}(\mathrm{CN})_{2}{ }^{-}$is $\mathrm{E}^{0}=$ $-0.6 \mathrm{~V}$ NHE. However, to the best of our knowledge, there are no references in the literature about the $\mathrm{TiO}_{2}$-assisted photocatalytic decomposition of gold-cyanide complexes. Serpone et al. (6) reported the only work in which gold was deposited from air-equilibrated solutions prepared by mixture of $\mathrm{NaCN}$ and $\mathrm{AuCl}_{3} \cdot 3 \mathrm{H}_{2} \mathrm{O}$. No trials for characterization of the gold-cyanide complexes that could be formed in the system were done. The goal of their work was to investigate the disposal of free $\mathrm{CN}^{-}$by oxidation with peroxides prior to irradiation of the solutions in the presence of $\mathrm{TiO}_{2}$. The fast oxidation of free cyanides avoided the formation of gold-cyanide complexes thus allowing $\mathrm{Au}(\mathrm{III})$ reduction on irradiated $\mathrm{TiO}_{2}$.

On the other hand, the photocatalytic processes have shown a high efficiency for the oxidation of free cyanides $(9,10)$, iron cyanocomplexes $(11,12)$ and copper cyanocomplexes (13).

In this work, a new procedure for gold recovery from solutions containing gold-cyanide complexes by using $\mathrm{TiO}_{2}-$ based semiconductor materials is reported. The photocatalytic treatment performed in the absence of oxygen leads to the reduction of gold to the metallic state and increases the availability of cyanide for subsequent oxidation treatment (14).

\footnotetext{
* Corresponding author
} 


\section{Fundamentals of the photocatalytic recovery of gold}

Figure 1 shows the fundamentals of the heterogeneous photocatalytic process (15) applied to the recovery of gold from cyanocomplexes in solution. When a semiconductor is illuminated with radiation whose energy is equal or higher than the bandgap of the material, electrons $\left(e^{-}\right)$are promoted to the conduction band, generating oxidant electron vacancies, so-called holes $\left(h^{+}\right)$in the valence band. These charge carriers can diffuse through the particle and reach the surface of the semiconductor particle. However, the lifetime of these species is really small since most of them usually recombine either in the bulk or at the particle surface releasing useless energy as heat. The photocatalytic reaction takes place when the charge carriers are transferred to chemical species adsorbed on the semiconductor.

The procedure described in this work for the photocatalytic recovery of gold from gold-cyanide complexes is based on their reduction by the photogenerated electrons. As a result, the deposition of metallic gold on the titania surface and the release from the complex of free cyanide ions take place. Simultaneously, in order to minimize the extension of the recombination processes in the semiconductor, an additional compound must be oxidized by the holes generated in the valence band. Such oxidation is usually mediated by the primary formation of hydroxyl radicals that are well-known strong oxidants (16). The optimal approach of the whole process for gold cyanocomplexes treatment would be that free cyanides accomplish the role of hydroxyl radical scavengers. In these conditions the simultaneous gold recovery and cyanide oxidation would be achieved. However, both processes can be inhibited by the presence in solution of other compounds that can compete for the charge carriers trapping.

The efficiency of a photochemical process is characterized by the quantum yield that represents the ratio between the moles of the target species converted per mole of photons absorbed by the system (17). Consequently, this parameter quantifies the relative extension of the successful charge carriers transfer and the recombination processes.

The use of the quantum yield in heterogeneous systems is not easy since it requires a precise measurement of the photons absorbed by the scattering medium. This problem can be overcome by referring the observed reaction rate to the incident flow of photons that enters into the system. This parameter is called photonic efficiency (17) and allows the comparison of different photocatalysts taking into account the losses of radiation scattered outside the system.

Finally, the assessment of the economics of the process needs an additional parameter: the energetic efficiency. It relates the conversion of the target compound to the

Table 1

Textural properties and $\mathrm{TiO}_{2}$ Crystal sizes of the catalysts

\begin{tabular}{|c|c|c|c|c|c|c|}
\hline \multirow[b]{3}{*}{ Catalyst } & \multirow{3}{*}{$\begin{array}{c}\text { BET surface } \\
\left(\mathrm{m}^{2} \mathrm{~g}^{-1}\right)\end{array}$} & \multirow{3}{*}{$\begin{array}{c}\text { Pore } \\
\text { volume } \\
\left(\mathrm{cm}^{3} \mathrm{~g}^{-1}\right)\end{array}$} & \multicolumn{4}{|c|}{$\mathrm{TiO}_{2}$ crystalline phases (XRD) } \\
\hline & & & \multicolumn{2}{|c|}{ Anatase } & \multicolumn{2}{|c|}{ Rutile } \\
\hline & & & $(\%)$ & Size $(\mathrm{nm})$ & $(\%)$ & Size $(\mathrm{nm})$ \\
\hline Degussa P25 & 52.0 & - & 80 & 31.0 & 20 & 54.0 \\
\hline $60 \% \mathrm{TiO}_{2} / \mathrm{GrSiO}_{2}$ & 179 & 0.67 & 100 & 12.2 & - & - \\
\hline
\end{tabular}

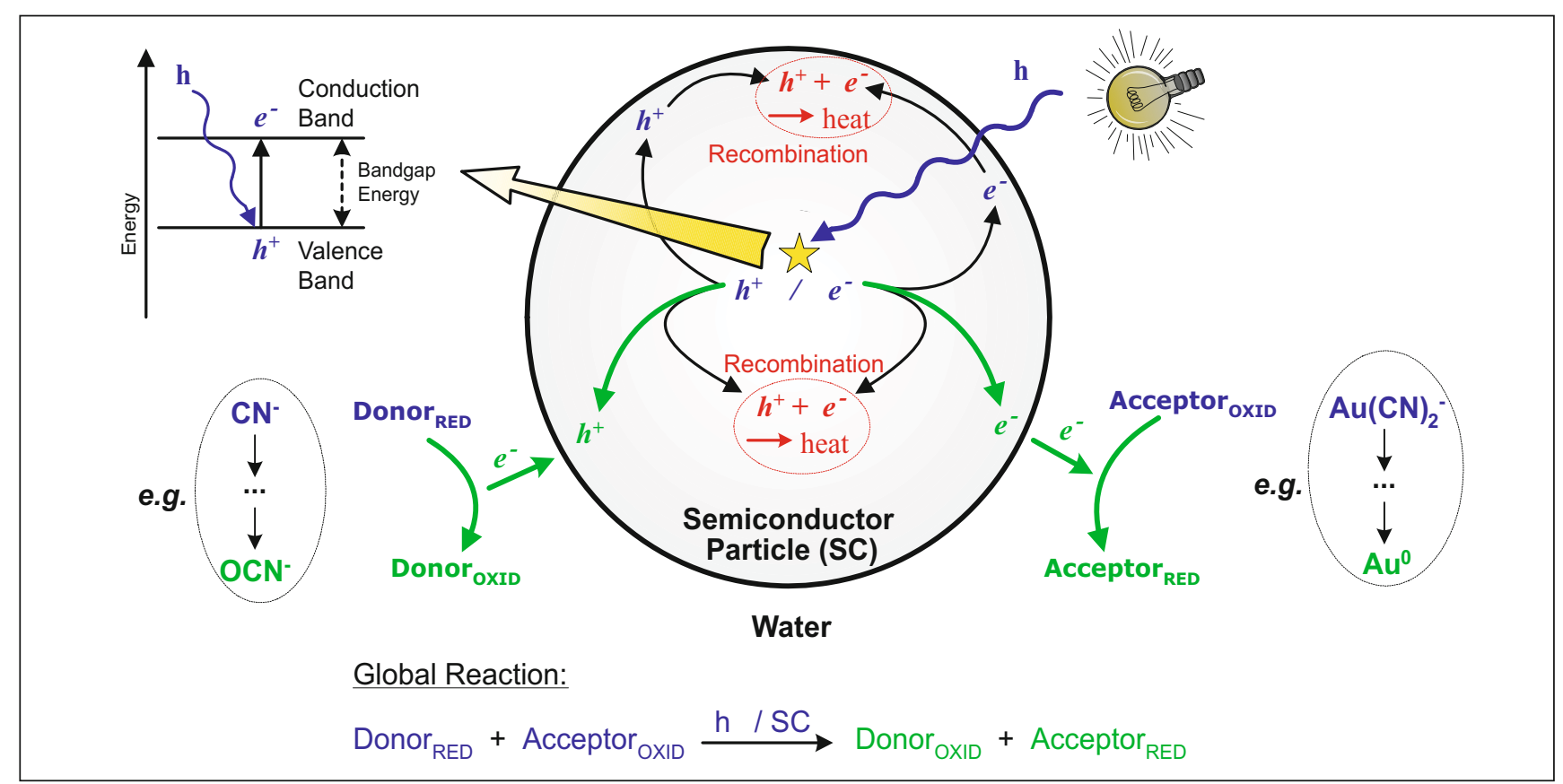

Figure 1

Fundamentals of the photocatalytic recovery of gold from cyanocomplexes 


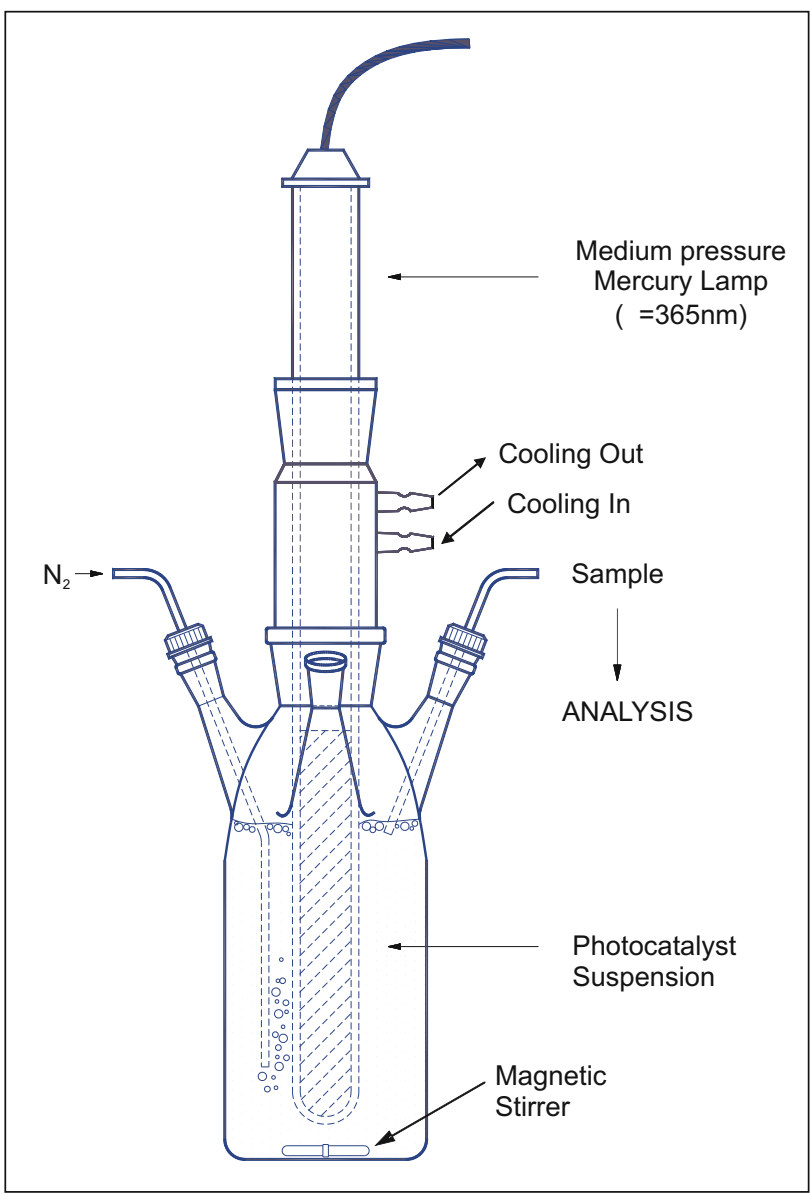

Figure 2

Schematic representation of the experimental setup

electrical energy required by the system (17), including the yield of the lamp generating the radiation and some additional aspects related to the reactor engineering.

\section{Catalysts}

Titanium dioxide is the semiconductor material most widely used in photocatalytic processes, due to its high stability against photocorrosion, adequate bandgap energy, null toxicity and low price. Among the materials commercially available, Degussa P25 is usually considered as the most active photocatalyst, and it has been proposed as standard for the comparison of the activity of different materials $(17,18)$. It consists of non-porous particles in the range of $30-$ 55 nanometers, with a B.E.T. specific surface area close to 50 $\mathrm{m}^{2} \mathrm{~g}^{-1}$. When this material is dispersed in water, bigger agglomerates of 0.3-0.9 micrometers are formed (19).

Unfortunately, for applications in aqueous phase such a small particle size means high filtration costs to remove the catalyst once the reaction is finished. These problems have motivated the development of supported photocatalysts in which $\mathrm{TiO}_{2}$ has been immobilized on diverse materials $(20,21)$. In addition to some other important properties, a suitable support must be chemically inert, transparent to the UV radiation and with a high specific surface area. Probably this is the reason why most of the work on immobilized photocatalysts has been focused to the use of siliceous materials as supports (20).

The $\mathrm{TiO}_{2} / \mathrm{SiO}_{2}$ photocatalyst used in this work was prepared by a sol-gel method, through the hydrolysis and condensation of titanium tetraisopropoxide inside the porous structure of a commercial silica support (Grace Sylopol 2104 $\mathrm{SiO}_{2}$ ). The titania content of this catalyst was $60 \mathrm{wt} \%$ and it will be referred to as $60 \% \mathrm{TiO}_{2} / \mathrm{CrSiO}_{2}$. X-ray diffraction patterns of the solid showed that anatase was the only crystalline phase. Table 1 summarises the main textural properties and titanium dioxide crystal sizes of both Degussa P25 $\mathrm{TiO}_{2}$ and the titania-silica photocatalyst. More details about the synthesis and characterization of these materials can be found elsewhere $(22,23)$.

\section{Photocatalytic reactions}

Experimental runs were carried out in a 1 litre Pyrex reactor with a $150 \mathrm{~W}$ medium pressure mercury lamp (Heraeus TQ150) placed inside and provided with a cooling tube. Figure 2 schematizes the experimental setup. An aqueous copper sulphate solution maintained at $25^{\circ} \mathrm{C}$ was circulated through the cooling tube to prevent overheating of the lamp and the solution and to avoid photolytic processes. The incident volumetric photon flow was $1.37 \cdot 10^{-5}$ Einstein $\mathrm{I}^{-1} \mathrm{~s}^{-1}$, determined by potassium ferrioxalate actinometry according to the method of Hatchard and Parker (24).

Photocatalytic reactions were carried out at $25^{\circ} \mathrm{C}$, with initial pH of 11.5 (adjusted with a sodium hydroxide solution) and catalyst concentration of $0.5 \mathrm{~g} \mathrm{TiO}_{2} \mathrm{l}^{-1}$. The catalyst was suspended in the solution by magnetic stirring. Depending on the conditions required for the reaction, either synthetic air or helium was bubbled through the suspension 30 minutes before starting the irradiation and throughout the reaction to ensure gas-equilibrated solutions. The lamp was switched on outside the reactor for 30 minutes before starting the irradiation of the solution in order to stabilize the emission spectrum and power.

In order to follow the evolution of the reaction, samples were taken through an opening in the upper part of the reactor vessel. Prior to the analysis, the catalyst was removed from the samples by using a nylon syringe filter of $0.22 \mu \mathrm{m}$.

Free cyanide concentrations were determined by a standard spectrophotometric method based on the blue-red colour development (absorption maximum at $575 \mathrm{~nm}$ ) upon addition of a pyridine-barbituric reagent (25). A Merck Spectroquant VEGA 400 spectrophotometer was used for the absorption measurements. Analysis of cyanide oxidation products (mainly cyanate and nitrate species) was carried out using a Metrohm ion chromatograph in an aqueous solution of $\mathrm{NaHCO}_{3}(2 \mu \mathrm{M})$ and $\mathrm{Na}_{2} \mathrm{CO}_{3}(1.3 \mu \mathrm{M})$ as eluent. The concentrations of soluble metallic species, mainly gold but also other different elements present in the spent platting baths, were determined by inductively coupled plasma atomic emission spectroscopy (ICP-AES) in a Varian Vista AX instrument. 


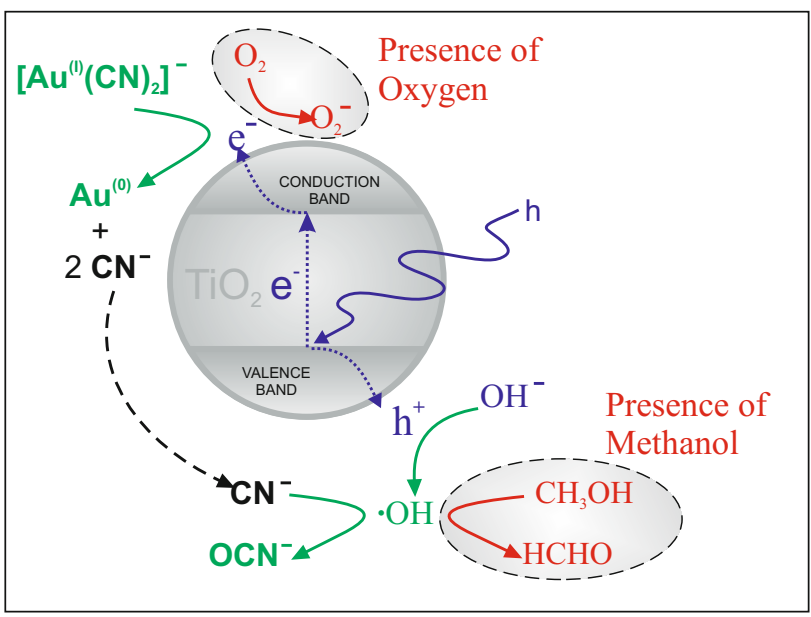

Figure 3

Possible reactions in the photocatalytic degradation of dicyanoaurate(I) in the presence of $\mathrm{O}_{2}$ and methanol as charge carriers scavengers

\section{Recovery of gold from pure dicyanoaurate(I) solutions using Degussa $\mathrm{P} 25 \mathrm{TiO}_{2}$}

Initially, experiments of photodegradation of pure $\mathrm{Au}(\mathrm{CN})_{2}$ solutions were carried out in order to investigate the kinetics and mechanism of the process prior to the study of solutions with more complex composition, such as real wastewater from plating facilities. Irradiation of the dicyanoaurate solutions without catalyst evidenced the absence of photolytic processes. On the other hand, no decrease of the dicyanoaurate concentration in solution by adsorption was observed after stirring a suspension of the complex and the catalyst after one hour in the dark.

Irradiation of $\mathrm{Au}(\mathrm{CN})_{2}$ - solutions in the presence of Degussa P25 $\mathrm{TiO}_{2}$ were carried out to check the feasibility of the photocatalytic dicyanoaurate complex degradation. The first series of experiments were performed in the presence of oxygen, but the results obtained showed no degradation of the gold complex. Some research groups have previously reported that the addition of organic compounds that act as hydroxyl radical scavengers could increase the photoreduction rate of metal species (26). However, this was not the case, since no degradation of the gold-cyanide complexes was observed even upon addition of methanol $(30 \mathrm{mM})$ as hydroxyl radical scavenger. These results are in agreement with the high stability of the gold-cyanide complexes observed by Serpone et al. (6).

Taking into account that molecular oxygen is a very good electron scavenger, lack of photocatalytic degradation of the dicyanoaurate in the presence of oxygen can be explained in terms of a competition between $\mathrm{O}_{2}$ and $\mathrm{Au}(\mathrm{CN})_{2}$ for conduction band electrons of the semiconductor. The more favourable reduction potential of molecular oxygen inhibits the reduction of $\mathrm{Au}(\mathrm{I})$. On the other hand, the added methanol competes with $\mathrm{CN}^{-}$ions for valence band holes (figure 3), avoiding OCN- formation. In fact, when methanol and oxygen are simultaneously present in solution, the photoxidation of methanol to formaldehyde with oxygen is

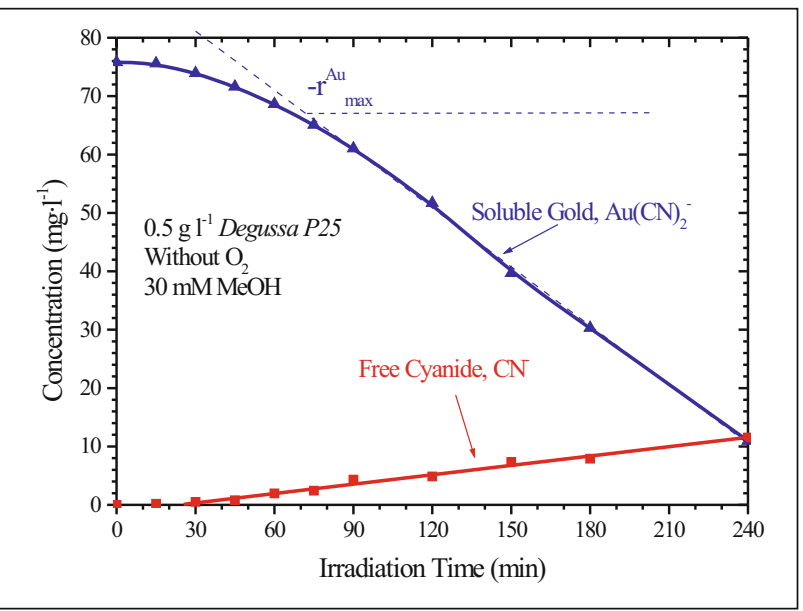

Figure 4

Photocatalytic gold deposition from dicyanoaurate-(I) with Degussa P25 $\mathrm{TiO}_{2}$

the only global reaction that takes place, and the gold cyanide complexes behave as inert compounds in solution.

Irradiation after helium bubbling caused no gold complex degradation, but successful gold deposition was obtained after methanol addition that promoted decomposition of the complex and release of free cyanide ions to the medium. Figure 4 shows the dicyanoaurate-(I) degradation profile observed in the photocatalytic treatment of a $75.8 \mathrm{mg}_{\mathrm{Au}} \mathrm{l}^{-1}$ solution using Degussa P25 $\mathrm{TiO}_{2}$, in absence of oxygen but with $30 \mathrm{mM}$ methanol added as hydroxyl radical scavenger. It is worth noting the increased availability of free cyanide released from the complex and the fact that it is not oxidized to cyanate in such conditions.

Figure 4 shows that gold deposition takes places after an initial delay. As no other electron acceptor is present except dicyanoaurate, this fact could be explained by the presence of oxygen traces that remained adsorbed on the $\mathrm{TiO}_{2}$ surface and whose reduction takes place prior to the reduction of dicyanoaurate ions. Another possible explanation is that once the deposition of metallic gold over the semiconductor surface starts, the charge transfer is improved due to the enhanced separation of the photogenerated electron-hole pairs induced by the presence of gold clusters on the semiconductor surface (27). A progressive increase of the reaction rate is observed up to a maximum value reached after 90 minutes, as it is shown in Figure 4. This maximum reduction rate ( $\mathrm{r}^{\mathrm{Au}}$ max $)$ will be the parameter used afterwards for the comparison of photocatalytic activities shown by bare $\mathrm{TiO}_{2}$ and $\mathrm{TiO}_{2} / \mathrm{SiO}_{2}$.

It is also worth noting that cyanide could be the hydroxyl radical scavenger required for the gold reduction in oxygen free solutions. However, as the release of cyanide from $\mathrm{Au}(\mathrm{CN})_{2}$ is coupled to the previous reduction of gold complexes, activation of the reaction requires additional hole scavengers such as methanol. In this case, taking into account that no cyanate formation was detected in any experiment, it must be assumed that the oxidation of released cyanide ions to cyanate species cannot be achieved in the system, despite the high activity previously shown by 
Table 2

Composition of the spent plating bath solutions

\begin{tabular}{|c|c|c|}
\hline \multirow[b]{2}{*}{ Component } & \multicolumn{2}{|c|}{ Concentration $\left(\mathrm{mg} \mathrm{l}^{-1}\right)$} \\
\hline & Bath 1 & Bath 2 \\
\hline Free cyanide & 16 & 0.5 \\
\hline Total organic carbon (TOC) & 6800 & 47000 \\
\hline Gold & 217 & 5760 \\
\hline Copper & 8 & 120000 \\
\hline Zinc & 120 & 146 \\
\hline Nickel & 168 & 24 \\
\hline Iron & 92 & 154 \\
\hline
\end{tabular}

$\mathrm{TiO}_{2}$ and $\mathrm{TiO}_{2} / \mathrm{SiO}_{2}$ for free cyanide photoxidation (23). The main reason is the presence of methanol, a more effective hydroxyl radical scavenger as compared with $\mathrm{CN}^{\text {; }}$, thus avoiding the reaction of $\mathrm{OH} \cdot$ with free cyanide anions. Nevertheless, it is important to point out that the decomposition of the metal complex is essential for further cyanide treatment because free $\mathrm{CN}^{-}$ions are readily available for a subsequent oxidation stage.

Consequently, the treatment of dicyanoaurate containing effluents must be carried out in two consecutive steps. In the first step the degradation of the metal complex must be performed in a non-oxidant atmosphere for gold deposition on $\mathrm{TiO}_{2}$ and the subsequent cyanide ions release to the solution. In the second step, oxidant conditions would be required in order to reach the conversion of free cyanide to cyanate.

\section{Recovery of gold from spent plating baths using Degussa P25 $\mathrm{TiO}_{2}$}

In order to evaluate the treatment of real industrial effluents, the photocatalytic degradation of two different spent plating baths was tested, whose composition is summarized in table 2. Both solutions show low concentration of free cyanide, as expected for their $\mathrm{pH}$ value (7.4 and 4.8 for bath 1 and 2, respectively), and high organic content, especially in bath 2 .

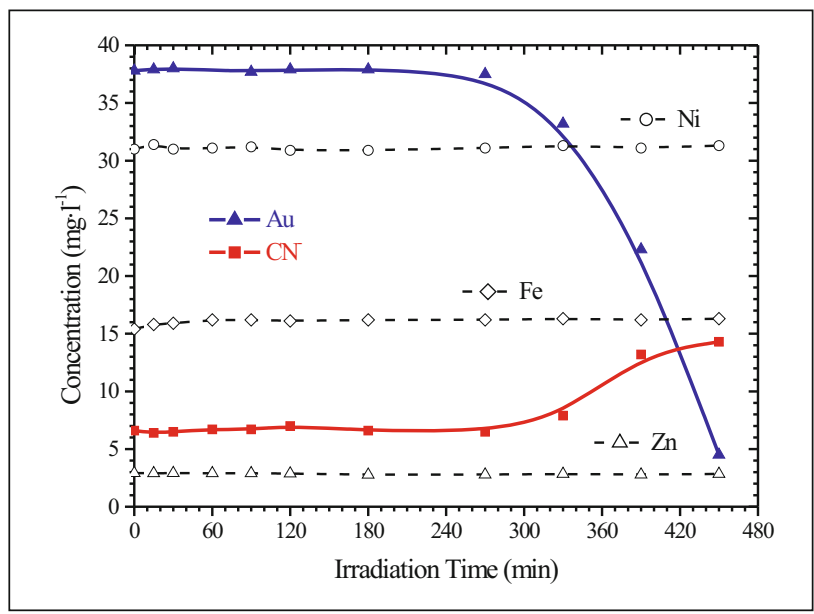

\section{Figure 5}

Photocatalytic gold deposition from bath 1 (dilution 1:5) with Degussa $\mathrm{P}_{25} \mathrm{TiO}_{2}$

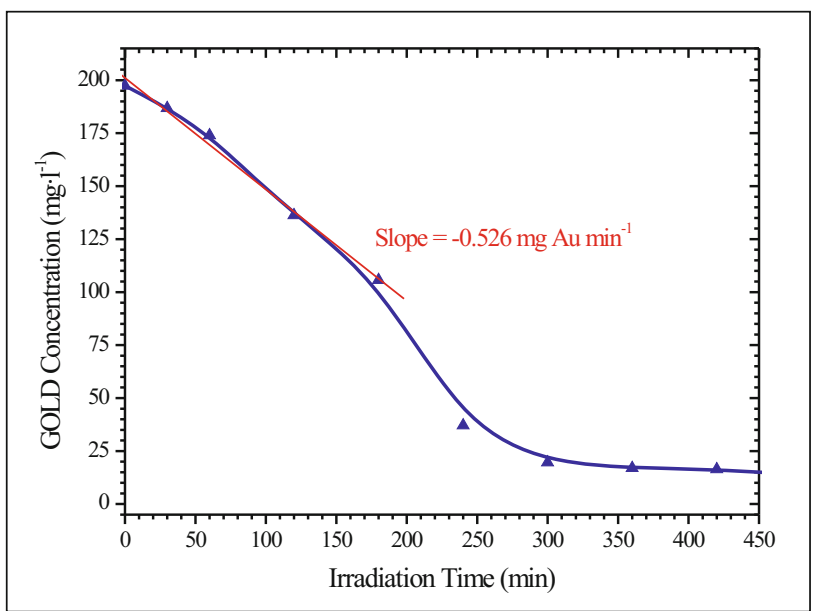

Figure 6

Photocatalytic gold deposition from bath 2 (dilution 1:30) with Degussa P25 $\mathrm{TiO}_{2}$

This fact is related to the use of additives such as brightening and complexing agents whose formulation belongs to the know-how of the company. But the most important feature to be mentioned is the extremely high concentration of copper in bath 2, suggesting that it was probably a mixed copper-gold plating solution. Finally, it is worth mentioning the presence of other heavy metals in solution, mainly nickel, zinc and iron.

The photocatalytic treatment of both effluents was carried out with and without methanol addition. The results obtained in both cases were very similar, suggesting that the organic matter initially present in the bath behaved as a proper hydroxyl radical scavenger as methanol does. Figure 5 shows the results obtained in the photodegradation of bath 1 (dilution 1:5) with Degussa P25 $\mathrm{TiO}_{2}$, where total recovery of gold could be achieved. It is worth noting the four hours delay observed before the beginning of the reduction process, much longer than in the case of the pure dicyanoaurate solutions, suggesting the existence of a reduction process more favourable than the decomposition of $\mathrm{Au}(\mathrm{CN})_{2}$. Analysis of the aqueous solutions by ICP-AES showed no variation in the concentration of nickel, zinc, iron

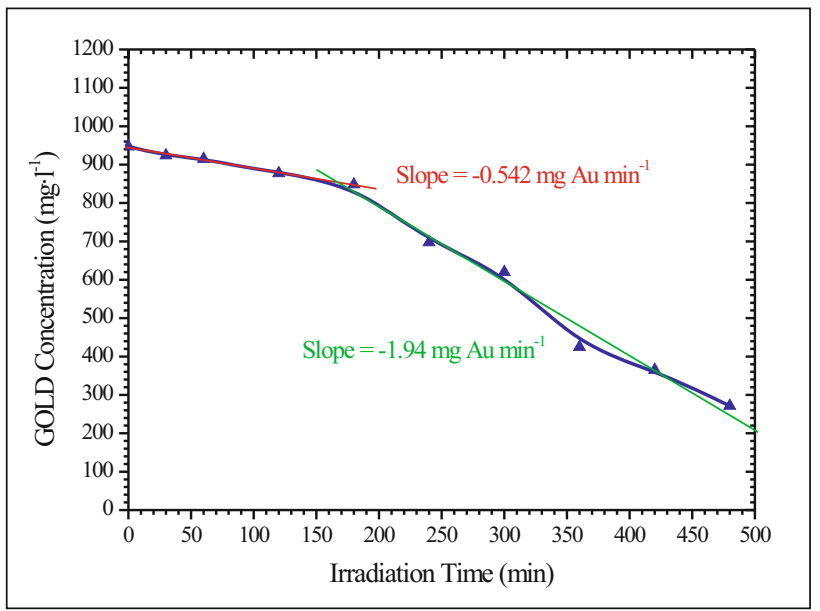

Figure 7

Photocatalytic gold deposition from bath 2 (dilution 1:6) with Degussa $\mathrm{P} 25 \mathrm{TiO}_{2}$ 


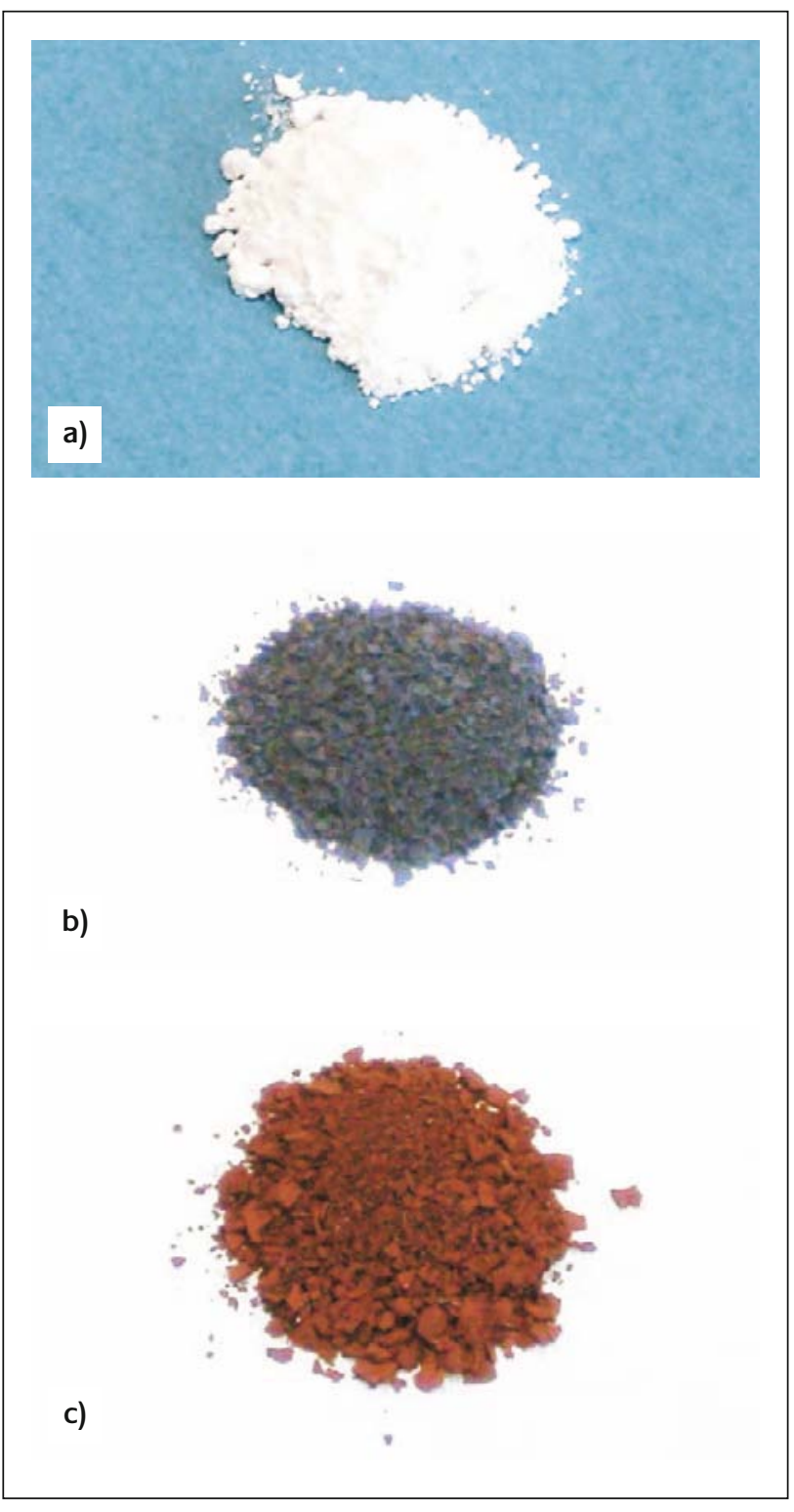

Figure 8

Photographs of the catalyst Degussa P25 (a), solid recovered after the reaction (b) and gold recovered from that solid (c)

and copper in the solution. Additionally, analysis of the solids showed no metals other than gold deposited on the semiconductor.

Concerning bath 2, figure 6 shows the results obtained in the photocatalytic treatment with Degussa P25. The initial delay is very similar to that obtained with pure dicyanoaurate solutions, which indicates that the delay observed in figure 5 is related to the specific composition of bath 1 .

As bath 2 presents a higher concentration in gold, an additional experiment was carried out starting from approximately $1 \mathrm{~g} \mathrm{l}^{-1}$ of gold solution. The results displayed in figure 7 show the existence of two different rates of deposition. In the first step, a deposition rate of 0.542 $\mathrm{mg}_{\mathrm{Au}} \mathrm{min}^{-1}$ is observed, essentially identical to that calculated from figure 6 . However, in this case, the recovery rate reaches a value close to $2 \mathrm{mg}_{\mathrm{Au}} \mathrm{min}^{-1}$ at higher reaction time. Consequently, the gold recovery rate is very dependent on the gold content and the specific composition of the bath.

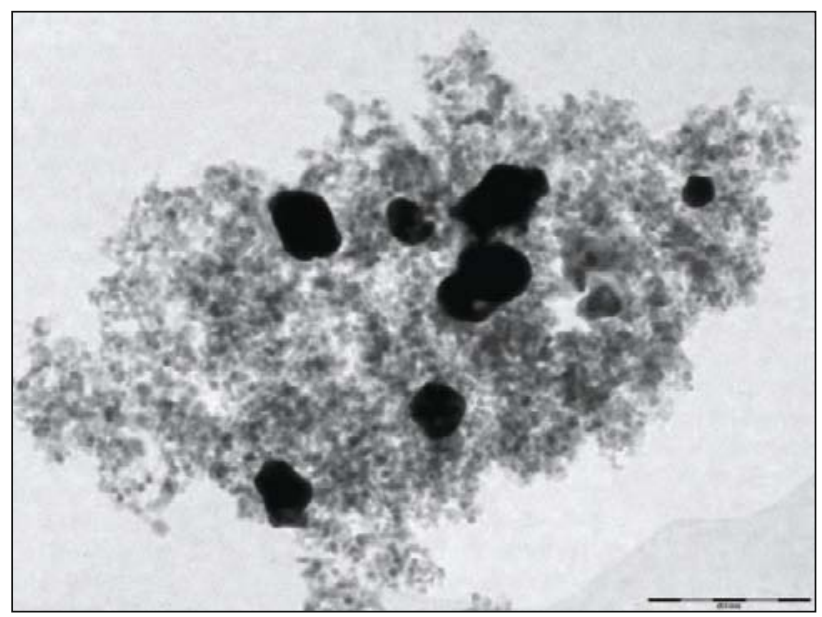

Figure 9

TEM micrograph of the solids recovered after reaction with Degussa $\mathrm{P} 25 \mathrm{TiO}_{2}$

\section{Final fate of gold}

In comparison with the white colour of the $\mathrm{TiO}_{2}$ catalyst, the solids recovered after the reaction showed an intense violet colour as it can be seen in figure 8 a) and b).

Analysis by ICP-AES of the solid recovered after the reaction showed that gold is the only metal deposited on the semiconductor. Determination of gold content confirmed the mass balance between gold deposition on the catalyst and cyanide disposal to the solution. A transmission electron micrograph of the solid is presented in figure 9. The microanalysis of the dark crystals with higher size than those of $\mathrm{TiO}_{2}$ showed that they are constituted by metallic gold.

Finally, the X-ray diffraction pattern, depicted in figure 10 , shows that in addition to the signals corresponding to the titanium dioxide crystalline phases, only metallic gold is detected.

Gold separation from the spent catalyst can be easily carried out by two different ways. Chemical attack with aqua regia produces the selective dissolution of gold as tetrachloroauric acid whereas the catalyst remains as a solid

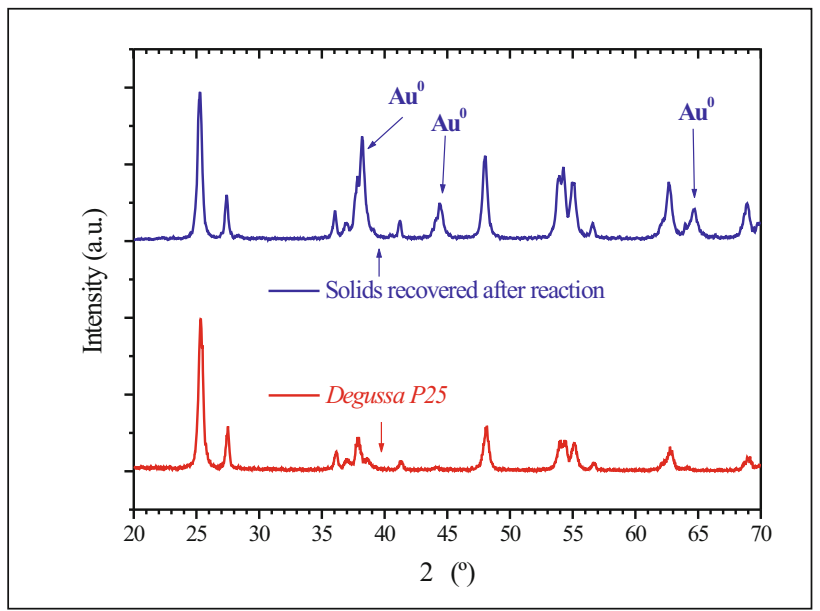

Figure 10

$X$-ray diffraction pattern of the solid recovered after the reaction with Degussa P25 $\mathrm{TiO}_{2}$ 
Table 3

Gold deposition rates and photonic efficiencies in the photocatalytic treatment of pure dicyanoaurate-(I) solutions

Catalyst $\quad-r^{\mathrm{Au}}{ }_{\max }\left(\mathrm{mg} \mathrm{Au} \mathrm{l}^{-1} \mathrm{~min}^{-1}\right) \quad \xi\left(\mathrm{mol}\right.$ Au Einstein $\left.{ }^{-1}\right)$

\begin{tabular}{lll}
\hline Degussa P25 & 0.336 & $3.83 \cdot 10^{-3}$ \\
\hline $60 \% \mathrm{TiO}_{2} / \mathrm{GrSiO}_{2}$ & 0.456 & $5.16 \cdot 10^{-3}$ \\
\hline
\end{tabular}

\section{Table 4}

Gold deposition rates and photonic efficiencies in the photocatalytic treatment of the spent plating bath solution number 1

\begin{tabular}{|c|c|c|}
\hline Catalyst & $r^{A u}{ }_{\max }\left(\mathrm{mg} \mathrm{Au} \mathrm{l}^{-1} \mathrm{~min}^{-1}\right)$ & $\xi\left(\mathrm{mol}\right.$ Au Einstein $\left.{ }^{-1}\right)$ \\
\hline Degussa P25 & 0.297 & $3.35 \cdot 10^{-3}$ \\
\hline $60 \% \mathrm{TiO}_{2} / \mathrm{GrSiO}_{2}$ & 0.373 & $4.22 \cdot 10^{-3}$ \\
\hline
\end{tabular}

phase. On the other hand chemical attack with hydrofluoric acid leads to the selective dissolution of the catalyst while gold remains as a brown powder (see figure $8, \mathrm{c}$ ) ready to be refined, if it is necessary. Obviously the first separation method allows the catalyst to be reused.

\section{Recovery of gold using the $\mathrm{TiO}_{2} / \mathrm{SiO}_{2}$ photocatalyst}

Similarly to the results obtained with bare $\mathrm{TiO}_{2}$, preliminary experiments of photodegradation of pure dicyanoaurate solutions with the $\mathrm{TiO}_{2} / \mathrm{SiO}_{2}$ photocatalyst showed that gold reduction only takes place if the gold complex is the only electron acceptor and a hydroxyl radical scavenger is added. Figure 11 displays the results obtained for the photocatalytic degradation of a pure $\mathrm{Ag}(\mathrm{CN})_{2}$ - solution and the spent plating bath number 1 with the catalyst $60 \% \mathrm{TiO}_{2} / \mathrm{GrSiO}_{2}$. In both cases, the results are analogous to those obtained with Degussa P25 $\mathrm{TiO}_{2}$.

Tables 3 and 4 show respectively the values of the gold deposition rates and the photonic efficiencies $(\xi)$, calculated as the ratio between the maximum gold reduction rate and the volumetric photon flow incident to the photoreactor. From the $\mathrm{r}_{\text {max }}^{\mathrm{Au}}$ values, the $\mathrm{TiO}_{2} / \mathrm{SiO}_{2}$ catalyst shows a degradation rate even higher than that obtained with the commercial $\mathrm{TiO}_{2}$ Degussa P25. Taking into account the higher activity shown by the $\mathrm{P} 25 \mathrm{TiO}_{2}$ for free cyanide photoxidation (23) these results suggest that in this case the silica surface improves the gold photoreduction due to adsorption phenomena and/or because of the higher specific surface area for gold deposition.

The values obtained for the photonic efficiencies are lower than those reported for other photocatalytic processes, typically over $10^{-2}$ mol Einstein-1 ${ }^{-1}$ In this case it can be considered as a minor drawback due to the recovery of such a valuable gold metal product. Anyway, improvements in the photonic efficiencies of the systems must be considered in order to increase the yield of the process.

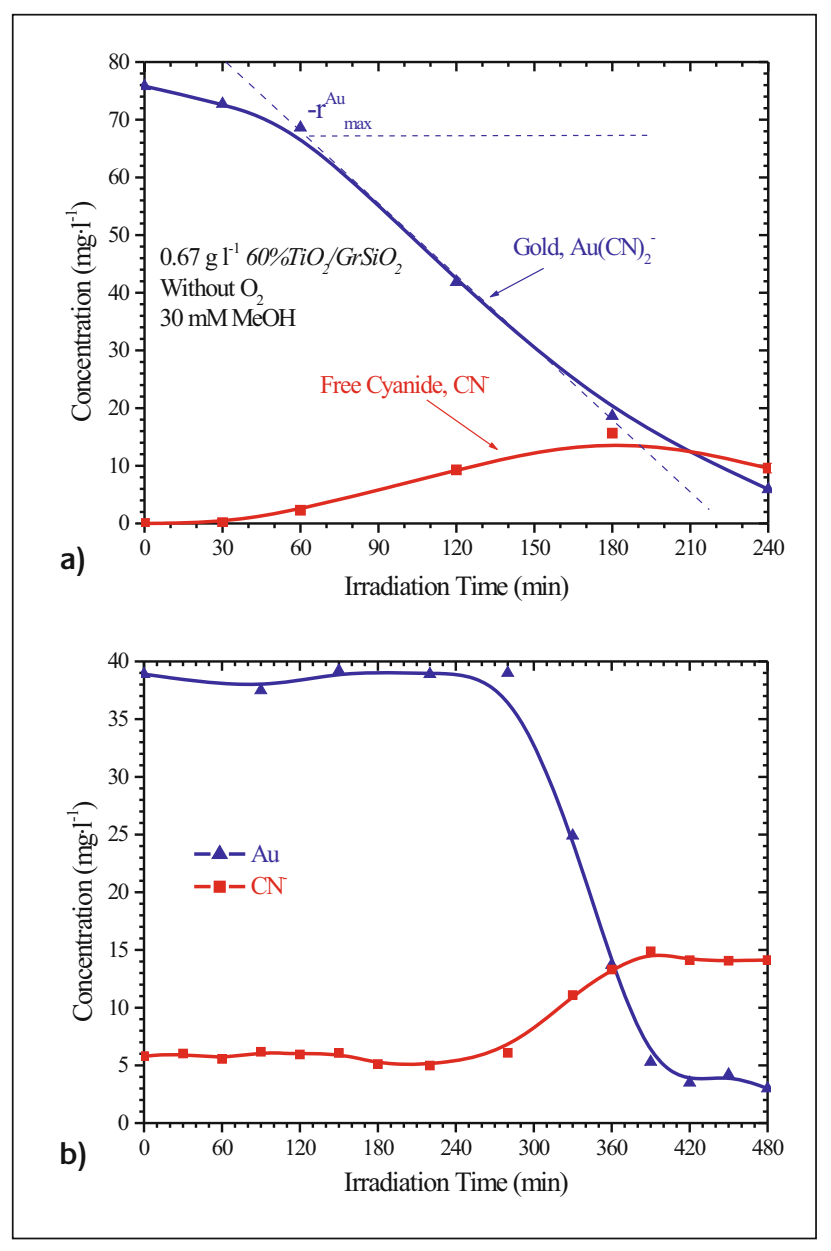

\section{Figure 11}

Photocatalytic gold deposition from pure dicyanoaurate (a) and from the bath 1 (b) using the catalyst 60\% $\mathrm{TiO}_{2} / \mathrm{GrSiO}_{2}$

\section{Conclusions}

A new photocatalytic procedure has been developed for the recovery of gold from cyanide complexes commonly occurring in electroplating works. It is worth mentioning that the use of titania-silica photocatalysts improved the gold deposition rate as compared with the treatment with bare $\mathrm{TiO}_{2}$. In addition to that, an increase in the availability of cyanide for the subsequent oxidation treatment is achieved due to the release of free cyanide ions from the stable metal cyanocomplexes.

Nevertheless, the reported results show that the performance of this procedure could be very dependent on the specific and complex composition of the electrolytic bath. Consequently, further investigation must be carried out in order to evaluate the feasibility of this process for the recovery of gold from these effluents.

\section{Acknowledgements}

This work was financially supported by Ministerio de Ciencia y Tecnología (PPQ2000-1287 project) and Consejería de Educación de la Comunidad de Madrid (Grupos Estratégicos de Investigación). Thanks are also due to D. Guillermo 
Zabaleta from Metalor Technologies Iberica and Montse Miernau from Cookson Electronics for the supply of the spent plating bath solutions.

\section{About the authors}

Dr. Rafael van Grieken is full professor of the Department of Chemical and Environmental Technology and Vicechancellor for Research and Development of Rey Juan Carlos University, in Móstoles (Madrid). He received his Ph.D. in Chemical Engineering at Complutense University in Madrid (Spain). His research interests are focused on the synthesis of catalytic materials and applications for several chemical processes from Photocatalytic wastewater treatment to Fine Chemicals production.

Dr. José Aguado is a full professor of the Department of Chemical and Environmental Technology and Director of the School of Experimental Sciences and Technology of the Rey Juan Carlos University, in Móstoles (Madrid). His research interest involves the synthesis of catalytic materials and their application to wastewater treatment, polymerization, chemical processes and chemical recycling of plastic wastes.

Dr. María José López Muñoz is an associate professor of the Department of Chemical and Environmental Technology of the Rey Juan Carlos University, in Móstoles (Madrid). She received her Ph.D. in Physical Chemistry at the Autónoma University in Madrid (Spain) in 1994. She was a visiting scientist at the Chemical Engineering Department in Palermo (Italy), the Chemistry Department in Bradford (United Kingdom) and the Institut de Recherches sur la Catalyse in Lyon (France). She was associate scientist at the Instituto de Catálisis y Petroleoquímica (C.S.I.C.) in Madrid (Spain). Her research interest includes the photocatalytic treatment of industrial effluents, the synthesis of photocatalysts and the spectroscopic characterization of photocatalytic systems.

Dr. Javier Marugán is an assistant professor of the Department of Chemical and Environmental Technology of the Rey Juan Carlos University, in Móstoles (Madrid). He received his Ph.D. in Chemical Engineering at Rey Juan Carlos University (2003), where his thesis research focuses on the development of silica-supported photocatalysts for the photocatalytic treatment of cyanide containing industrial wastewater. He was a visiting scientist at the Institut für Technische Chemie (ITC) of the Hannover University, Germany, and the Instituto de Desarrollo Tecnológico para la Industria Química (INTEC) of Santa Fé, Argentina (CONICET-Universidad del Litoral). His research interest involves the photocatalytic treatment of industrial effluents.

\section{References}

1 "Gold, gold alloys and gold compounds", "Cyano Compounds, Inorganic" and "Electrochemical and chemical deposition" in Ullman's Encyclopedia of Industrial Chemistry, 6th edition, Wiley-VCH, Weinheim, Germany, 1998

2 R.J. Irwin, M. van Mouwerik, L. Stevens, M.D. Seese and W. Basham, “Environmental Contaminants Encyclopedia: Cyanide”. National Park Service, Water Resources Division, Fort Collins, Colorado, 1997

3 C.A. Young and T.S. Jordan, "Cyanide remediation: Current and past technologies". Proceedings of the 10th Annual Conference on Hazardous Waste Research, Kansas State University, Manhattan, Kansas. May 23-24, 1995, 104

4 E. Borgarello, R. Harris and N. Serpone, Nouv. J. Chim., 1985, 9, 743

5 E. Borgarello, N. Serpone, G. Emo, R. Harris, E. Pellizzetti and C. Minero, Inorg. Chem., 1986, 25, 4499

6 N. Serpone, E. Borgarello, M. Barbeni, E. Pelizzetti, P. Pichat, J.M. Hermann and M.A. Fox, J. Photochem., 1987, 36, 373

7 M. Albert, Y.M. Gao, D. Toft, K. Dwight and A. Wold, Mat. Res. Bull., 1992, 27, 961

8 C.Y. Wang, C.Y. Liu, X. Zheng, J. Chen and T. Shen, Colloids Surf. A: Phys. Eng. Aspects, 1998, 131, 271

9 S.N. Frank and A.J. Bard, J. Amer. Chem. Soc., 1977, 99, 303

10 V. Augugliaro, V. Loddo, G. Marcì, L. Palmisano and López-Muñoz, M.J., J. Catal., 1997, 166, 272

11 W.S. Rader, L. Solujic, E.B. Milosavljevic and J.L. Hendrix, Environ. Sci. Technol., 1993, 27, 1875

12 R. van Grieken, J. Aguado, M.J. López-Muñoz and J. Marugán, Appl. Catal B. Environ., 2005, 55, 201

13 J. Peral and X. Domenech, J. Chem. Tech. Biotechnol., 1992, 53, 93

14 J. Aguado, R. van Grieken, M.J. López-Muñoz and J. Marugán, PCT Pat. Appl., PCT/ES 2004/000283

15 M.R. Hoffmann, S.T. Martin, W. Choi and D.W. Bahnemann, Chem. Rev., 1995, 95, 69

16 C.S. Turchi and D.F. Ollis, J. Catal., 1990, 122, 178

17 N. Serpone and A. Salinaro, Pure \& Appl. Chem., 1999, 71, 303

18 A. Salinaro, A.V. Emeline, J. Zhao, H. Hidaka, V.K. Ryabchuk and N. Serpone, Pure \& Appl. Chem., 1999, 71, 321

19 M.I. Cabrera, O.M. Alfano and A.E. Cassano, J. Phys. Chem., 1996, 100, 20043

20 R.L. Pozzo, M.A. Baltanás and A.E. Cassano, Catal. Today, 1997, 39, 219

21 J.A. Byrne, B.R. Eggins, N.M.D. Brown, B. McKinney and M. Rouse, Appl. Catal. B: Environ., 1998, 17, 25

22 R. van Grieken, J. Aguado, M.J. López-Muñoz and J. Marugán, J. Photochem. Photobiol. A: Chem., 2002, 148, 315

23 J. Aguado, R. van Grieken, M.J. López-Muñoz and J. Marugán, Catal. Today, 2002, 75, 95

24 C.G. Hatchard and C.A. Parker, Proc. Roy. Soc. (London), 1956, 518

25 L.S. Clescerl, A.E. Greenberg and A.D. Eaton, (Eds). "Standard Methods for the Examination of Water and Wastewater" p. 4-33, United Book Press, Inc., Baltimore, Maryland, United States, 1998

26 D. Chen and A.K. Ray, Chem. Eng. Sci., 2001, 56, 1561

27 M. Jakob, H. Levanon and P.V. Kamat, Nano Lett., 2003, 3, 353 